\title{
Afetos positivos e negativos em estudantes de graduação em Enfermagem
}

\author{
Positive and negative affects in undergraduate Nursing students \\ Afectos positivos y negativos en estudiantes de licenciatura en Enfermería
}

Recebido: 03/03/2021 | Revisado: 09/03/2021 | Aceito: 12/03/2021 | Publicado: 20/03/2021

Daniel Augusto da Silva
ORCID: https://orcid.org/0000-0002-2716-6700
Fundação Educacional do Município de Assis, Brasil
E-mail: daniel.augusto@unifesp.br
João Emanuel Ribeiro Santos
ORCID: https://orcid.org/0000-0002-2501-5765
Fundação Educacional do Município de Assis, Brasil
E-mail: joao.emanuelribeiro83@gmail.com
Lisley Galvão Lucio
ORCID: https://orcid.org/0000-0002-0682-8330
Fundação Educacional do Município de Assis, Brasil
E-mail: 1starja@yahoo.com.br
Ana Paula Teixeira Rosa
ORCID: https://orcid.org/0000-0001-8443-8998
Fundação Educacional do Município de Assis, Brasil
E-mail: paularosa.mk@hotmail.com
Elizete Mello da Silva
ORCID: https://orcid.org/0000-0002-6606-5291
Fundação Educacional do Município de Assis, Brasil
E-mail: dedemelo@femanet.com.br
Rosângela Gonçalves da Silva
ORCID: https://orcid.org/0000-0002-3223-750X
Fundação Educacional do Município de Assis, Brasil
E-mail: roseziquinelli@gmail.com
Caroline Lourenço de Almeida

\section{Resumo}

Objetivo: compreender os afetos positivos e afetos negativos vivenciados por estudantes de graduação em Enfermagem. Métodos: Trata-se de estudo transversal, epidemiológico, quantitativo, com 59 estudantes de graduação em Enfermagem, matriculados em instituição de ensino superior de cidade no centro-oeste do estado de São Paulo. Os dados foram coletados entre os meses de setembro e outubro de 2018, por meio de questionário semiestruturado, elaborado pelos autores, para caracterização dos participantes quanto as variáveis sociodemográficas e aplicação da Escala de Afetos Positivos e Negativos. Os dados foram analisados com estatística descritiva e análise inferencial ANOVA um fator, tendo como variável dependente a intensidade de experimentação de cada afeto, e como preditores as variáveis sociodemográficas. O nível de significância adotado para as análises deste estudo foi de $\mathrm{p}<0,05$. Resultados: Os participantes apresentaram média geral de 4,3 e moda 3,8 para intensidade de afetos positivos, e média geral de 3,4 e moda 3,2 para intensidade de afetos negativos. $\mathrm{Na}$ análise dos afetos positivos, evidenciou-se maior intensidade de afetos positivos em heterossexuais $(\mathrm{p}=0,012)$; presença de filhos $(\mathrm{p}=0,035)$; e ausência de transtornos mentais $(\mathrm{p}=0,006)$. Aos afetos negativos, evidenciou-se maior intensidade de afetos negativos na presença de transtornos mentais $(\mathrm{p}=0,003)$. Considerações finais: Os afetos positivos são mais presentes que os afetos negativos em estudantes de graduação em Enfermagem. Essa situação pode estar relacionada aos sentimentos de entusiasmo, realização e expectativa quanto ao futuro e melhoria das condições de vida sonhadas com o ingresso no ensino superior. Palavras-chave: Enfermagem; Estudantes de enfermagem; Educação superior; Emoções.

\begin{abstract}
Objective: to understand the positive and negative affects experienced by undergraduate nursing students. Methods: This is a cross-sectional, epidemiological, quantitative study, with 59 undergraduate nursing students, enrolled in a higher education institution in a city in the central-west of the state of São Paulo. Data were collected between the months of September and October 2018, through a semi-structured questionnaire, prepared by the authors, to characterize the participants regarding the sociodemographic variables and the application of the Positive and Negative Affections Scale. The data were analyzed using descriptive statistics and inferential ANOVA one factor, with the intensity of experimentation of each affect as a dependent variable, and sociodemographic variables as predictors. The
\end{abstract}


level of significance adopted for the analyzes of this study was $\mathrm{p}<0.05$. Results: The participants had a general average of 4.3 and a mode of 3.8 for the intensity of positive affects, and a general average of 3.4 and a mode of 3.2 for intensity of negative affects. In the analysis of positive affects, there was a greater intensity of positive affects in heterosexuals $(p=0.012)$; presence of children $(p=0.035)$; and absence of mental disorders $(p=0.006)$. Negative affects showed a higher intensity of negative affects in the presence of mental disorders $(\mathrm{p}=0.003)$. Final considerations: Positive affects are more present than negative affects in undergraduate nursing students. This situation may be related to feelings of enthusiasm, fulfillment and expectations about the future and improvement in the living conditions dreamed of entering higher education.

Keywords: Nursing; Nursing students; College education; Emotions.

\section{Resumen}

Objetivo: comprender los afectos positivos y negativos que experimentan los estudiantes de licenciatura en enfermería. Métodos: se trata de un estudio transversal, epidemiológico, cuantitativo, con 59 estudiantes de licenciatura en enfermería, matriculados en una institución de educación superior de una ciudad del centro-oeste del estado de São Paulo. Los datos fueron recolectados entre los meses de septiembre y octubre de 2018, a través de un cuestionario semiestructurado, elaborado por los autores, para caracterizar a los participantes en cuanto a las variables sociodemográficas y la aplicación de la Escala de Afectos Positivos y Negativos. Los datos se analizaron mediante estadística descriptiva y análisis ANOVA inferencial con un factor, con la intensidad de experimentación de cada afecto como variable dependiente y las variables sociodemográficas como predictoras. El nivel de significancia adoptado para los análisis de este estudio fue $\mathrm{p}<0.05$. Resultados: Los participantes presentaron un promedio general de 4.3 y una moda de 3.8 para la intensidad de afectos positivos, y una media general de 3.4 y una moda de 3.2 para la intensidad de afectos negativos. En el análisis de afectos positivos, hubo mayor intensidad de afectos positivos en heterosexuales ( $\mathrm{p}$ $=0,012)$; presencia de hijos $(p=0,035)$; y ausencia de trastornos mentales $(p=0,006)$. Los afectos negativos mostraron una mayor intensidad de afectos negativos en presencia de trastornos mentales $(p=0,003)$. Consideraciones finales: Los afectos positivos están más presentes que los negativos en los estudiantes de enfermería. Esta situación puede estar relacionada con sentimientos de entusiasmo, realización y expectativas sobre el futuro y la mejora en las condiciones de vida soñadas al ingresar a la educación superior.

Palabras clave: Enfermería; Estudiantes de enfermería; Educación universitaria; Emociones.

\section{Introdução}

A vida universitária carregada inúmeras experiências e transformações na vida dos estudantes, Como experiências positivas desses jovens cita-se a transição para a vida adulta, com a desejada independência ao sair da casa dos pais, esperança de construção de um futuro melhor e orgulho, contudo, há experiências negativas, como a necessidade de adaptação a tarefas secundárias à vida escolar, a sobrecarga emocional e o estresse, relacionado a momentos de mudança, desenvolvimento, frustração, crescimento, temores e angústias (Cardoso, Gomes, Pereira Junior, \& Silva, 2019; Vizzotto, Jesus, \& Martins, 2017).

O estilo de vida do estudante universitário pode ser influenciado pelo ambiente universitário e as experiências vividas nele, com adoção de práticas saudáveis ou prejudiciais à saúde. Realidades cognitivas e emocionais por vezes intrínsecas a este ambiente, que outrora é local desejado por muitos jovens, pois é entendido como a única forma de ascensão social e melhoria das condições de vida (Berbigier \& Magalhães, 2017; Silva \& Marcolan, 2015; Silva, 2019).

Ao analisar as experiências e práticas de vida adotadas por universitários, deve-se considerar as influências na dimensão afetiva, que são construídas a partir das vivências acadêmicas, de modo que experiências positivas geram emoções positivas, e, da mesma forma, experiências negativas geram emoções negativas (Matta, Lebrao, \& Heleno, 2017; Anjos \& Aguilar-da-Silva, 2017).

Em outras palavras, essas experiências são influenciadas por um estado emocional transitório, constituído de afetos positivos e afetos negativos, de modo que afetos positivos culminam em sentimentos agradáveis, e, na outra extremidade, ansiedade, aborrecimento, pessimismo e angústia perfazem os afetos negativos, emoções desagradáveis e indesejáveis (Silva \& Heleno, 2012).

Dessa forma, evidencia-se a importância de estudos que abordem a saúde mental dos estudantes universitários, com foco na compreensão das experiências vividas e de como os mesmos foram afetados, pois, este conhecimento, é promissor para o emprego de ações que promovam a saúde e previnam doenças e agravos em decorrência da vida universitária. 
Nesse contexto, o objetivo do presente estudo foi compreender os afetos positivos e afetos negativos vivenciados por estudantes de graduação em Enfermagem.

\section{Metodologia}

Trata-se de estudo transversal, epidemiológico, quantitativo (Pereira, Shitsuka, Parreira, \& Shitsuka, 2018), com 59 estudantes de graduação em Enfermagem, matriculados em instituição de ensino superior de cidade no centro-oeste do estado de São Paulo.

Optou-se por um modelo de amostragem não probabilística por conveniência, de modo que todos os alunos que atendessem aos critérios de inclusão foram considerados a população do estudo. Os critérios de inclusão compreenderam ser aluno regularmente matriculado na instituição, estar presente na sala de aula no dia elegido para coleta dos dados e consentir com a participação voluntária.

Os dados foram coletados entre os meses de setembro e outubro de 2018, por meio de questionário semiestruturado, elaborado pelos autores, para caracterização dos participantes quanto as variáveis sociodemográficas e aplicação da Escala de Afetos Positivos e Negativos, composta por dez adjetivos que indicam estados afetivos, cinco positivos (feliz, alegre, satisfeito, divertido e otimista) e cinco negativos (deprimido, preocupado, frustrado, raivoso e infeliz). Emprega-se uma escala tipo Likert de sete pontos que varia de 1 a 7 ( 1 = nada, $2=$ muito pouco, $3=$ pouco, $4=$ mais ou menos, $5=$ bastante, $6=$ muito, $7=$ extremamente) que quantificam a intensidade de experimentação de cada afeto (Diener, Emmons, Larsen, \& Griffin, 1985).

Os participantes deste estudo dedicaram, em média, dez minutos para responder aos questionários.

Os dados foram analisados com estatística descritiva e análise inferencial ANOVA um fator, tendo como variável dependente a intensidade de experimentação de cada afeto, e como preditores as variáveis sociodemográficas. O nível de significância adotado para as análises deste estudo foi de $\mathrm{p}<0,05$.

Todos os participantes deste estudo assinaram o Termo de Consentimento Livre e Esclarecido, concordando a participação e somente após responderam ao questionário respectivo a esta pesquisa, respeitando a legislação específica para pesquisas com seres humanos.

O projeto foi submetido ao Comitê de Ética em Pesquisa da Fundação Educacional do Município de Assis (FEMA), sob CAAE 92564218.9.0000.8547 e obteve aprovação sob parecer nº 2.895.995, de 14 de setembro de 2018.

\section{Resultados}

Este estudo obteve a participação voluntária de 59 (48,0\%) estudantes universitários de graduação em Enfermagem, de 123 (100,0\%) possíveis, que apresentaram média geral de 4,3 e moda 3,8 para intensidade de afetos positivos, e média geral de 3,4 e moda 3,2 para intensidade de afetos negativos. Considerando a possibilidade de pontuação entre 1 (nada) e 7 (extremamente), é possível afirmar que os participantes revelaram a vivência de afetos positivos e negativos em diferentes níveis de intensidade (Figura 1). 
Figura 1. Boxplot de comparação de intensidade de afetos positivos e afetos negativos em estudantes universitários de Enfermagem ( $\mathrm{n}=59)$. Assis, (SP), Brasil, 2021.

\section{Intensidade de afetos positivos e negativos em estudantes de Enfermagem}

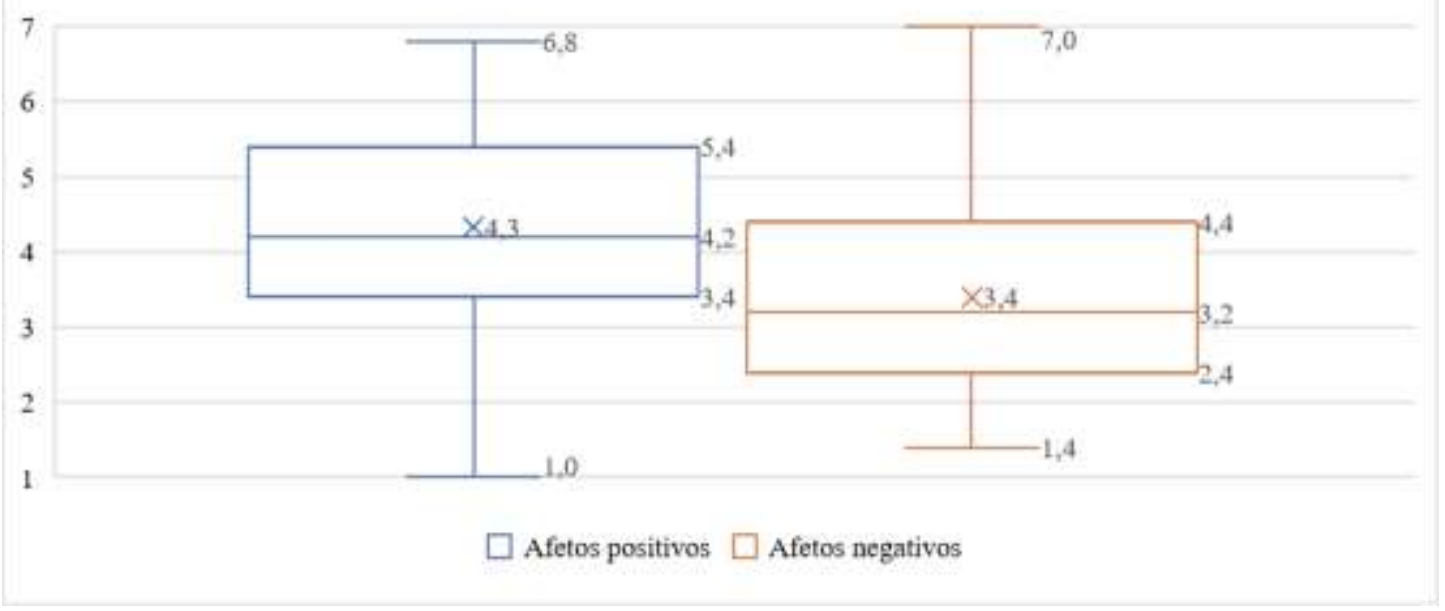

Fonte: Autores.

Observa-se que a intensidade de afetos positivos variou de 1,0 a 6,8, fato que permite a afirmação da existência de experimentação próximo ao máximo, classificado como extremamente, e a inexistência de afetos positivos, com a avaliação mínima possível $(1,0=$ nada). Em termos gerais, a maioria dos participantes apresentam intensidade de afetos positivos entre 3,4 e 4,2 (quartil 2). A descrição detalhada da intensidade de experiência de cada afeto positivo está demonstrada na Figura 2.

Figura 2. Boxplot de comparação de intensidade de cada afeto positivo em estudantes universitários de Enfermagem $(\mathrm{n}=59)$. Assis, (SP), Brasil, 2021.

\section{Afetos positivos em estudantes de Enfermagem}

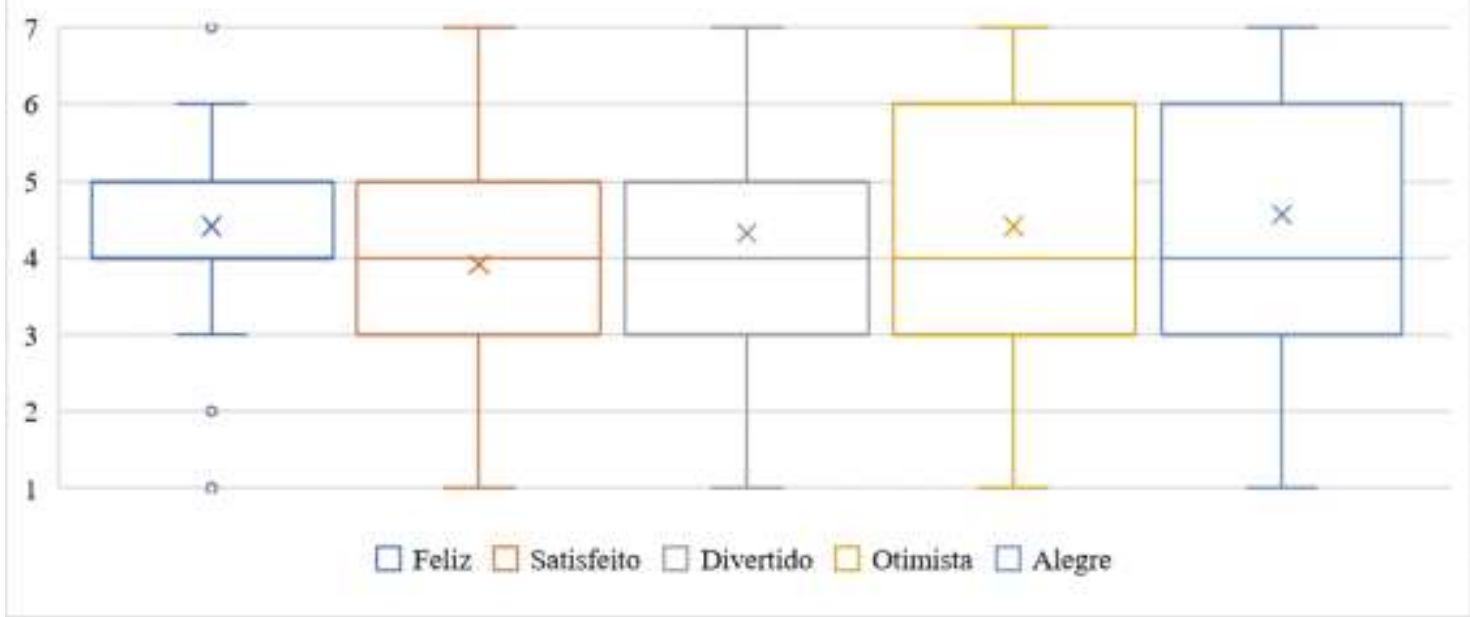

Fonte: Autores.

Aos afetos negativos, conforme a Figura 1, a intensidade de afetos negativos variou de 1,4 a 7,0, fato que permite a afirmação da existência de experimentação próximo ao mínimo, e a existência de afetos negativos de forma extrema. Em termos 
gerais, a maioria dos participantes apresentam intensidade de afetos positivos entre 2,4 e 3,2 (quartil 2). A descrição detalhada da intensidade de experiência de cada afeto negativo está demonstrada na Figura 3, de forma que se destaca a maior intensidade de preocupação frente aos demais afetos negativos.

Figura 3. Boxplot de comparação de intensidade de cada afeto negativo em estudantes universitários de Enfermagem $(\mathrm{n}=59)$. Assis, (SP), Brasil, 2020.

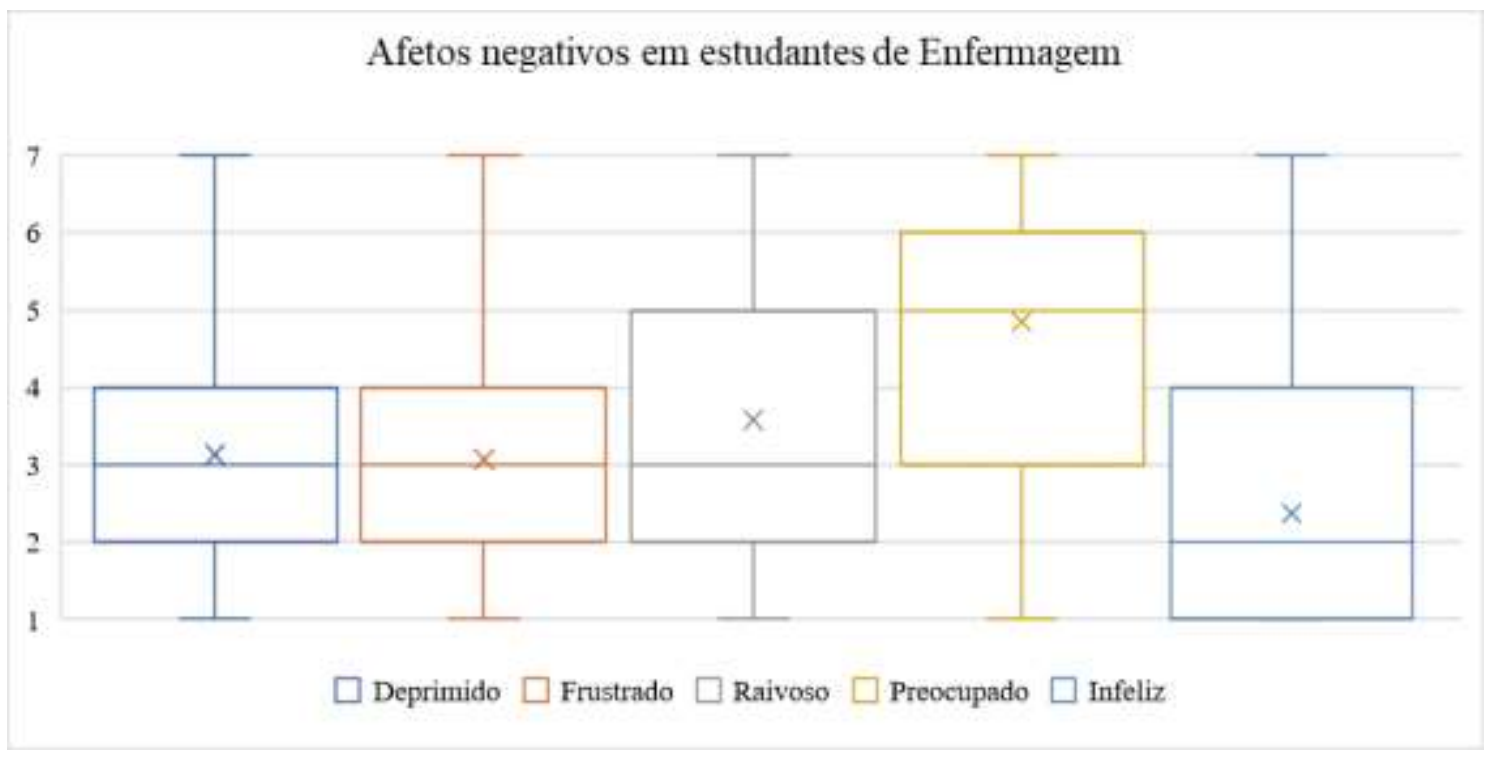

Fonte: Autores.

$\mathrm{Na}$ caracterização dos participantes, associados a avaliação da intensidade de afetos positivos e negativos, observa-se maior participação de mulheres $(91,5 \%)$, nas quais a média de afetos positivos foi menor $(4,2)$ do que em homens $(5,2)$, e média de afetos negativos maior $(3,5)$ do que em homens $(2,8)$. Outras informações sobre a caracterização sociodemográfica dos participantes e a avaliação da intensidade de afetos positivos e negativos estão descritas na Tabela 1.

$\mathrm{Na}$ análise dos afetos positivos, evidenciou-se diferença estatisticamente significante em relação as seguintes variáveis: orientação sexual $(\mathrm{p}=0,012)$ com maior intensidade de afetos positivos em heterossexuais; presença de filhos $(\mathrm{p}=0,035)$ com maior intensidade de afetos positivos na presença de filhos; e diagnóstico de transtornos mentais $(\mathrm{p}=0,006)$ com maior intensidade de afetos positivos na ausência de transtornos mentais. Dessa forma, é possível afirmar a diferença de comportamento entre esses grupos.

Quanto a análise da intensidade de afetos negativos, evidenciou-se diferença estatisticamente significante em relação ao diagnóstico de transtornos mentais $(\mathrm{p}=0,003)$ com maior intensidade de afetos negativos na presença de transtornos mentais. Ainda, destaca-se que em relação a orientação sexual, há tendência para diferença de comportamento entre os grupos (heterossexuais, homossexuais e bissexuais), com $\mathrm{p}=0,061$. 
Tabela 1. Associação entre as variáveis sociodemográficas e os afetos positivos e negativos em estudantes universitários de Enfermagem ( $\mathrm{n}=59)$. Assis, (SP), Brasil, 2021.

\begin{tabular}{|c|c|c|c|c|c|c|c|}
\hline \multirow{2}{*}{ Variáveis (n) } & \multirow{2}{*}{ n $(\%)$} & \multicolumn{2}{|c|}{ Afetos Positivos } & \multirow{2}{*}{ p-Valor* } & \multicolumn{2}{|c|}{ Afetos Negativos } & \multirow{2}{*}{ p-Valor* } \\
\hline & & Média & Variação & & Média & Variação & \\
\hline SEXO & & & & 0,125 & & & 0,542 \\
\hline Feminino & $54(91,5)$ & 4,2 & $1,0-6,8$ & & 3,5 & $1,4-7,0$ & \\
\hline Masculino & $5(8,5)$ & 5,2 & $4,2-6,6$ & & 2,8 & $1,6-5,2$ & \\
\hline FAIXA ETÁRIA & & & & 0,251 & & & 0,253 \\
\hline Jovem (até 19 anos) & $16(27,1)$ & 4,1 & $2,6-6,4$ & & 3,8 & $1,4-5,8$ & \\
\hline Adulto (20 a 50 anos) & $43(72,9)$ & 4,4 & $1,0-6,8$ & & 3,3 & $1,4-7,0$ & \\
\hline ORIENTAÇÃO SEXUAL & & & & $\mathbf{0 , 0 1 2}$ & & & 0,061 \\
\hline Heterossexual & $56(94,9)$ & 4,4 & $2,6-6,8$ & & 3,3 & $1,4-5,8$ & \\
\hline Homossexual & $2(3,4)$ & 3,4 & $2,0-4,8$ & & 4,7 & $2,4-7,0$ & \\
\hline Bissexual & $1(1,7)$ & 1,0 & & & 6,2 & & \\
\hline FILHOS & & & & $\mathbf{0 , 0 3 5}$ & & & 0,475 \\
\hline Não & $48(84,1)$ & 4,2 & $1,0-6,6$ & & 3,5 & $1,4-7,0$ & \\
\hline Sim & $11(18,6)$ & 5,0 & $3,0-6,8$ & & 3,1 & $1,6-5,2$ & \\
\hline RELIGIÃO & & & & 0,836 & & & 0,827 \\
\hline Sim & $55(93,2)$ & 4,3 & $1,0-6,8$ & & 3,4 & $1,4-7,0$ & \\
\hline Não & $4(6,8)$ & 4,5 & $4,0-5,0$ & & 3,4 & $2,6-5,2$ & \\
\hline PATOLOGIA FÍSICA & & & & 0,448 & & & 0,121 \\
\hline Não & $53(89,8)$ & 4,4 & $1,0-6,8$ & & 3,3 & $1,4-7,0$ & \\
\hline Sim & $6(10,2)$ & 3,8 & $2,6-4,8$ & & 4,1 & $2,4-5,8$ & \\
\hline TRANSTORNO MENTAL & & & & 0,006 & & & 0,003 \\
\hline Não & $51(86,4)$ & 4,5 & $2,6-6,8$ & & 3,2 & $1,4-5,8$ & \\
\hline Sim & $8(13,6)$ & 3,2 & $1,0-5,0$ & & 4,7 & $2,4-7,0$ & \\
\hline $\begin{array}{l}\text { ESTUDANTE } \\
\text { TRABALHADOR }\end{array}$ & & & & 0,729 & & & 0,714 \\
\hline Sim & $34(57,6)$ & 4,2 & $2,0-6,8$ & & 3,5 & $1,4-7,0$ & \\
\hline Não & $25(42,4)$ & 4,4 & $1,0-6,4$ & & 3,3 & $1,4-6,2$ & \\
\hline
\end{tabular}

Fonte: Autores.

\section{Discussão}

O presente estudo buscou compreender os afetos positivos e afetos negativos vivenciados por estudantes de graduação em Enfermagem. A sensação e julgamento sobre si e sobre o outro é induzida um estado de ânimo que pode ser positivo ou negativo, o afeto (Noronha, Lamas, \& Barros, 2016).

A análise individual acerca do meio social vivido é influenciada pelos afetos. Ao perceber afetos positivos, as experiências carregarão prazer nas atividades, mesmo que sejam tarefas árduas, enquanto que afetos negativos influenciam o olhar negativo e triste da situação, tendo o desprazer como sensação marcante àquela atividade (Alves \& Ambiel, 2018).

Os afetos positivos constituem os componentes do bem estar positivo, de modo que refletem a percepção favorável da vida, o prazer e a felicidade. Ao vivenciar um afeto positivo, a pessoa comporta-se com entusiasmo, de forma ativa e autoconfiante. O desempenho nas atividades é marcado pela eficiência, concentração e energia (Alves \& Ambiel, 2018; Parente, Cunha, Galhardo, \& Couto, 2018).

$\mathrm{Na}$ outra ponta, o afeto negativo se relaciona ao desconforto, que por sua vez culmina em angústia e desprazer. Inclui emoções desagradáveis, como raiva, medo, culpa e desgosto. O desempenho nas atividades é marcado pela letargia e desprezo, e até aversão (Alves \& Ambiel, 2018; Parente, Cunha, Galhardo, \& Couto, 2018).

A satisfação de estudantes de ensino superior é marcada pelas percepções acerca das experiências vividas no contexto universitário, que engloba "o acesso e utilização dos espaços, equipamentos e recursos da instituição, a aprendizagem e rendimento académico, a organização do curso e ensino dos professores, as relações com os colegas e os serviços de apoio" (Almeida, Taveira, Peixoto, Silva, \& Gouveia, 2020).

Apesar da ciência sobre os múltiplos desafios enfrentados com o ingresso no ensino superior, e que estes desafios 
ameaçam o bem-estar pessoal, cognitivo, profissional, afetivo e social desses estudantes universitários, interferindo positivamente ou negativamente na formação (Ribeiro, Silveira, \& Teixeira, 2017), o presente estudo apresenta que os afetos positivos sobressaíram aos afetos negativos.

Ao compreender que afetos positivos se inclinam ao prazer, conforto e entusiasmo nas atividades e experiências, e que o ensino superior é entendido, por muitos, como a única forma de inserção no mercado de trabalho, este pode estar produzindo sensação de entusiasmo, realização e expectativa quanto ao futuro e melhoria das condições de vida (Berbigier \& Magalhães, 2017; Noronha \& Mansão, 2012), e, ainda, pode ser entendida como instrumento de medida de qualidade do ensino e fornecimento de apoio às necessidades dos estudantes (Almeida, Taveira, Peixoto, Silva, \& Gouveia, 2020).

A vivência de afetos positivos é uma necessidade no contexto acadêmico, pois interferem na construção de relações sociais consistentes e melhor formação acadêmica. Nas experiências positivas, destaca-se as habilidades sociais, que apresentam potencial para o senso de competência e pró atividade, com trocas positivas e maduras entre colegas e professores (Sorares, Buscacio, Fernandes, Medeiros, \& Monteiro, 2017).

Contudo, os afetos negativos também estão presentes, e a literatura afirma os altos níveis de estresse que estudantes de saúde estão expostos, e que, ainda, podem resultar em malefícios psicológicos, como a autocrítica e baixa autoestima, e físicos, como a síndrome do intestino irritável, angústia e a depressão (Araujo, Santana, Kazasa, Lacerda, \& Tanaka, 2020).

Enfatiza-se que níveis elevados de afetos negativos indicam a maior frequência e intensidade de experiências desagradáveis e de desprazer, e pessoas nessa condição consideram-se tristes, desanimadas e preocupadas (Noronha, Martins, Campos, \& Mansão, 2015; Zanon, Bastianello, Pacico, \& Hutz, 2013).

Neste contexto, é necessário esclarecer que a sensação de bem-estar, produzida por afetos, está relacionada aos traços de personalidade e às características sociodemográficas, de modo que a felicidade está relacionada a saúde, a educação e ao perfil extrovertido e otimista (Noronha, Martins, Campos, \& Mansão, 2015; Frenhan \& Silva, 2021). Essas condições podem alterar a percepção de uma mesma situação, por pessoas diferentes.

\section{Considerações Finais}

Os afetos positivos são mais presentes que os afetos negativos em estudantes de graduação em Enfermagem. Essa situação pode estar relacionada aos sentimentos de entusiasmo, realização e expectativa quanto ao futuro e melhoria das condições de vida sonhadas com o ingresso no ensino superior.

Sugerimos que futuros estudos se dediquem a analisar as interferências dos fatores pessoais, como os traços de personalidade, na percepção das situações e, consequentemente, do afeto produzido.

\section{Agradecimentos}

Programa de Iniciação Científica da Fundação Educacional do Município de Assis.

\section{Referências}

Almeida, L. S., Taveir,a M. C., Peixoto, F., Silva, J. C., \& Gouveia, M. J. (2020). Escala de satisfação no domínio académico em universitários portugueses. Revista Iberoamericana de Diagnóstico y Evaluación e Avaliação Psicológica, 54(1), 93-101. http://www.doi.org/10.21865/RIDEP54.1.08

Alves, B. P. \& Ambiel, R. A. M. (2018). Escala de forças de caráter: relações com instrumentos de avaliação de afetos e interesses profissionais. Estudos Interdisciplinares em Psicologia, 9(2), 4-20. http://www.doi.org/10.5433/2236-6407.2016v9n1p4

Anjos, D. R. L., \& Aguilar-da-Silva, R. H. (2017). Questionário de Vivências Acadêmicas (QVA-R): avaliação de estudantes de medicina em um curso com currículo inovador. Avaliação, 22(1), 105-123. http://www.doi.org/10.1590/S1414-40772017000100006

Araujo, A. C., Santana, C. L., Kazasa, E. H., Lacerda, S. S., \& Tanaka, L. H. (2020). Efeitos de um curso de meditação de atenção plena em estudantes da saúde no Brasil. Acta Paul Enferm, 33, 1-9. http://www.doi.org/10.37689/actaape/2020AO0170 
Berbigier, M. C., \& Magalhães, C. R. (2017). Educação nutricional em universitários e estratégias para promoção de saúde institucional: revisão integrativa. Rev Bras Promoç Saúde, 30(2), 264-274. http://www.doi.org/10.5020/18061230.2017.p264

Cardoso, J. V., Gomes, C. F. M., Pereira Junior, R. J., \& Silva, D. A. (2019). Estresse em estudantes universitários: uma abordagem epidemiológica. Rev enferm UFPE on line, 13, e241547 http://www.doi.org/10.5205/1981-8963.2019.241547

Diener, E., Emmons, R. A., Larsen, R., \& Griffin, S. (1985). The satisfaction with Life scale. Journal of Personality Assessment, 49(1), 71-75. http://www.doi.org/10.1207/s15327752jpa4901_13

Frenhan, M. \& Silva, D. A. (2021). A felicidade na voz de estudantes universitários. Research, Society and Development, 10(2), e27610212505. http://www.doi.org/10.33448/rsd-v10i2.12505

Matta, C. M. B., Lebrao, S. M. G., \& Heleno, M. G. V. (2017). Adaptação, rendimento, evasão e vivências acadêmicas no ensino superior: revisão da literatura. Psicol. Esc. Educ, 21(3), 583-591. http://www.doi.org/10.1590/2175-353920170213111118

Noronha, A. P. P., \& Mansão, C. S. M. (2012). Interesses profissionais e afetos positivos e negativos: estudo exploratório com estudantes de ensino médio. Psico-USF, 17(2), 323-331.

Noronha, A. P. P., Martins, D. F., Campos, R. R. F., \& Mansão, C. S. M. (2015). Relações entre afetos positivos e negativos e os cinco fatores de personalidade. Estudos de Psicologia, 20(2), 92-101. http://www.doi.org/10.5935/1678-4669.20150011

Noronha, A. P. P., Lamas, K. C. A., \& Barros, M. V. C. (2016). Afetos e personalidade: suas relações em estudantes universitários. Revista Psicologia: Teoria e Prática, 18(2), 75-88. http://www.doi.org/10.15348/1980-6906/psicologia.v18n2p75-88

Parente, L., Cunha, M., Galhardo, A., \& Couto, M. (2018). Autocompaixão, bem-estar subjetivo e estado de saúde na idade avançada. Revista Portuguesa de Investigação Comportamental e Social, 4(1), 3-13. http://www.doi.org/10.7342/ismt.rpics.2018.4.1.57

Pereira, A. S., Shitsuka, D. M., Parreira, F. J., \& Shitsuka, R. (2018). Metodologia da pesquisa científica. UFSM.

Ribeiro, I. K. S., Silveira, R. C. P., \& Teixeira, G. S. (2017). Estilo de vida y bienestar de estudiantes del área de la salud. Revista Enfermería Actual, 34, 1-16.

Silva, D. A., \& Marcolan, J. F. (2015). Desempleo y sufrimiento psíquico en enfermeras. Rev. Bras. Enferm, 68(5), 775-782. http://www.doi.org/10.1590/0034$7167.2015680502 \mathrm{i}$

Silva, D. A. (2019). A autoestima e o comportamento suicida em estudantes universitários: uma revisão da literatura. Revista Eletrônica Acervo Saúde, 23, e422. http://www.doi.org/10.25248/reas.e422.2019

Silva, E. C., \& Heleno, M. G. V. (2012). Qualidade de vida e bem-estar subjetivo de estudantes universitários. Revista Psicologia e Saúde, 4(1):69-76.

Sorares, A. B., Buscacio, R. C. Z., Fernandes, A. M., Medeiros, H. C. P., \& Monteiro, M. C. (2017). O impacto dos comportamentos sociais acadêmicos nas habilidades sociais de estudantes. Gerais: Revista Interinstitucional de Psicologia, 10(1), 69-80.

Vizzotto, M. M., Jesus, S. N., \& Martins, A. C. (2017). Saudades de casa: indicativos de depressão, ansiedade, qualidade de vida e adaptação de estudantes universitários. Rev. Psicol. Saúde; 9(1), 59-73. http://www.doi.org/10.20435/pssa.v9i1.469

Zanon, C., Bastianello, M. R., Pacico, J. C., \& Hutz, C. S. (2013). Desenvolvimento e validação de uma escala de afetos positivos e negativos. Psico-USF, 18(2), 193-202. http://www.doi.org/10.1590/S1413-82712013000200003 